\title{
ULTRASOUND EVIDENCE OF ACUTE APPENDICITIS IN A PATIENT WITH HEMOLYTIC UREMIC
} SYNDROME

\author{
Rija Siddiqui*, Shahid Nadeem*, Neethu Menon*, Jo-Ann Nesiama* \\ * University of Texas Southwestern Medical Center, Pediatric Emergency Medicine, Dallas, United \\ States
}

doi.org/10.33706/jemcr.543056

\begin{abstract}
Introduction: Hemolytic uremic syndrome (HUS) is a clinical syndrome characterized by microangiopathic hemolytic anemia, thrombocytopenia, and acute kidney injury. Children with HUS often present with gastrointestinal complaints, including abdominal pain, vomiting, and diarrhea. This presentation can mimic other gastrointestinal illnesses, including appendicitis, which further adds to the known diagnostic challenge.

Case Report: We report an 8-year-old male who presents with abdominal pain and diarrheaassociated HUS. Kidney ultrasound obtained for acute kidney failure showed an incidental finding of a dilated appendix which was confirmed on a follow-up appendix ultrasound. Patient lacked the typical findings of acute appendicitis such as fever, abdominal tenderness or rigidity. We observed the patient without medical or surgical treatment for acute appendicitis as ultrasound findings were attributed to HUS, and the patient's abdominal pain and HUS resolved without any intervention.

Conclusion: Self-limited appendiceal inflammation may be seen in diarrhea-associated HUS, and the diagnostic utility of appendix ultrasound to evaluate for acute appendicitis may be limited in a patient with HUS and abdominal pain.
\end{abstract}

Keywords: Hemolytic uremic syndrome; appendicitis; microangiopathic hemolytic anemia

\section{Introduction}

Children with diarrhea-associated HUS often present with symptoms of colitis such as abdominal pain, vomiting, and diarrhea, which can mimic a myriad of other common gastrointestinal processes, including gastroenteritis, appendicitis (1), intussusception, and inflammatory bowel disease. In addition, patients with HUS are at risk for life-threatening gastrointestinal complications such as bowel necrosis and perforation.

\section{Case Report}

An 8-year-old Caucasian male with a past medical history of asthma and seasonal allergies, presented to the emergency department (ED) with a one-week history of intermittent, generalized abdominal pain, pallor and weakness. Ten days prior to ED presentation, patient had diarrhea, nonbloody, non-bilious vomiting, and fever, which lasted a few days. On further review of systems, patient had yellowish skin, but negative for blood in the stool or urine, decreased urine output, or rash. Vital signs were normal apart from slight tachycardia to 120 beats/minute. Physical examination 
revealed an afebrile and tired-looking boy, a soft abdomen with mild guarding and splenomegaly on abdominal palpation, but no tenderness or rigidity. Pallor and jaundice was noted on skin examination. Laboratory evaluation was remarkable for leukocytosis (white blood cell count 15,700 $/ \mathrm{mm} 3$ ), normocytic anemia (hemoglobin $4.7 \mathrm{~g} / \mathrm{dL}$ ), mild thrombocytopenia (platelets $135,000 / \mathrm{mm} 3$ ), and kidney failure (blood urea nitrogen $36 \mathrm{mg} / \mathrm{dL}$, serum creatinine 1.0 $\mathrm{mg} / \mathrm{dL}$ ). Peripheral blood smear showed evidence of microangiopathic hemolytic anemia in the form of schistocytes and polychromasia (Figure 1). Hemolysis was also supported with the presence of hyperbilirubinemia (total serum bilirubin 2 $\mathrm{mg} / \mathrm{dL}$ ), reticulocytosis (16.5\%), and low haptoglobin $(<31 \mathrm{mg} / \mathrm{dL})$. Urinalysis revealed large occult blood, 2+ protein, and 6 red blood cells. Fecal occult blood was negative. Due to acute kidney injury, a renal ultrasound was performed, which revealed no anatomic kidney abnormality, but incidental findings of acute appendicitis. This was followed up by a dedicated appendix ultrasound, which showed an appendix $8 \mathrm{~mm}$ in maximal outer diameter with surrounding inflammation and hyperemia (Figure 2). The patient was admitted to the hospital, and nephrology, hematology, and general surgery services were consulted.

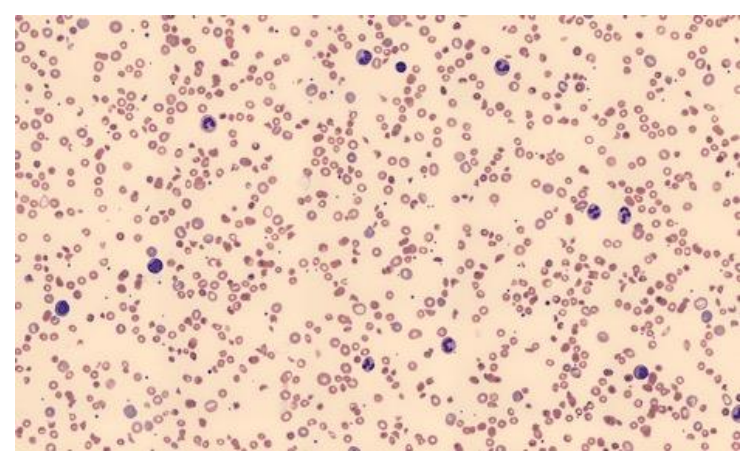

FIGURE 1. Peripheral blood smear showed evidence of microangiopathic hemolytic anemia in the form of schistocytes and polychromasia

The differential diagnoses included HUS (typical versus atypical), acute appendicitis, and thrombotic thrombocytopenic purpura (TTP). The patient received a $15 \mathrm{~mL} / \mathrm{kg}$ packed red blood cell transfusion for severe and symptomatic anemia with close monitoring for volume overload.

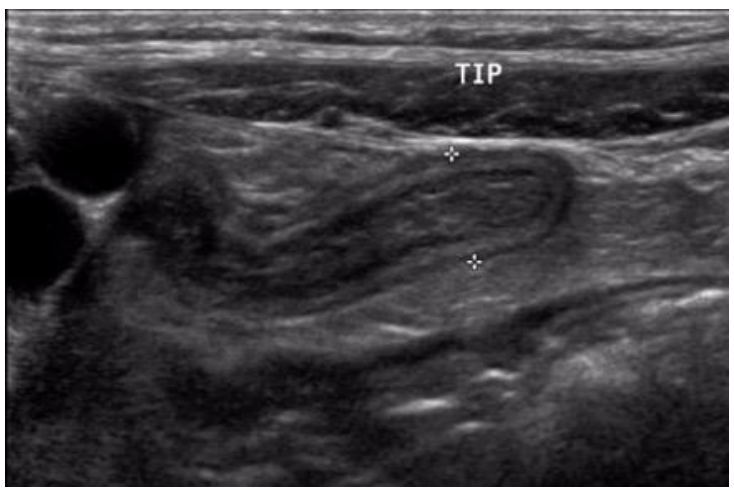

FIGURE 2. Appendix ultrasound, which showed an appendix $8 \mathrm{~mm}$ in maximal outer diameter with surrounding inflammation and hyperemia anemia in the form of schistocytes and polychromasia

Acute appendicitis was considered unlikely due to a consistent benign abdominal examination and absence of fever. As patient met the criteria for HUS by the simultaneous occurrence of microangiopathic hemolytic anemia, thrombocytopenia, and acute kidney injury, the abnormal appendix ultrasound findings were attributed to inflammatory processes secondary to HUS, and the patient did not receive antibiotics or undergo appendectomy. The patient had no further episodes of abdominal pain during admission. Renal function tests improved with normal saline bolus $(20 \mathrm{~mL} / \mathrm{kg}$ ) followed by intravenous and oral rehydration. Patient's urine output remained normal and he did not require renal replacement therapy. Complement studies (C3, C4) were within normal limits and atypical HUS genetic panel was negative. Subsequently, the patient was diagnosed with typical or diarrhea-positive HUS. Stool culture was not performed due to lack of stool specimen. At nephrology followup 6 months later, the patient was doing well with no lasting sequelae.

\section{Discussion}

Due to our patient's positive ultrasound findings for acute appendicitis, we were faced with a diagnostic dilemma to differentiate primary appendicitis from secondary appendicitis due to HUS. A literature review highlights a case from 1978 where the diagnosis of HUS was confused with acute appendicitis (2), which eventually resulted in 
a 2-year-old undergoing unnecessary appendectomy. There is a known clinical parallel between the HUS prodrome and appendicitis; in a 1973 review of 28 cases of typical or diarrhea positive HUS, $100 \%$ of patients had gastrointestinal symptoms, $46 \%$ had abdominal pain, and 2 patients underwent emergent appendectomy prior to the correct diagnosis of HUS (3). The review does not comment on abdominal imaging for the two patients who underwent appendectomy.

However, as a 1991 study suggests, appendectomies in these situations of diagnostic confusion may not yield typical results; in four cases where unnecessary appendectomy was performed, the operative findings were not typical of primary acute appendicitis, although two of these cases did have histological evidence of inflammation of the appendix (4). The study did not comment on imaging prior to appendectomy but did report that these patients did not have typical symptoms of appendicitis. Another case illustrates the rarity of HUS followed by appendicitis (5), where a patient with diagnosed HUS developed an acute change in abdominal examination prompting ultrasound imaging, which ultimately revealed acute appendicitis. This same patient at presentation 5 days earlier had a CT scan illustrating pancolitis without appendicitis. However, our literature search did not find any cases of ultrasound evidence of acute appendicitis at initial presentation in a patient with presumed HUS. Additionally, as our patient did not necessitate medical or surgical intervention for the ultrasound findings of appendicitis, this may limit the diagnostic utility of ultrasound in a patient with HUS and abdominal pain.

HUS, a thrombotic microangiopathy, can be classified as typical (associated with Shiga toxin producing Escherichia Coli infection), atypical (associated with uncontrolled complement activation), or secondary HUS (associated with a coexisting disease)(6). Although a stool sample was not obtained to document the presence of Shiga-toxin producing bacterial strains, our patient fits into the category of typical or diarrhea associated HUS due to presence of diarrhea, normal serum complements, and spontaneous resolution of symptoms. Shiga toxin in intestinal epithelial cells is associated with the production of pro-inflammatory, thrombogenic, and chemotactic mediators, which results in tissue injury (7). The complement system also plays an important role in typical HUS and infections can lead to deregulation of this system (8). Although gastrointestinal involvement with HUS has been reported in the colon, pancreas, liver, gall bladder, and esophagus (9), appendiceal involvement appears to be rare. We presume that our patient's appendix ultrasound findings were likely secondary to Shiga-like toxin mediated inflammation and that in HUS, endothelial cells of the appendix may be affected similarly to the colon. Although our patient did not have repeat appendix imaging, we can presume that due to the self-limited nature of HUS and our patient's clinical improvement, his appendiceal inflammation also resolved.

\section{Conclusion}

Our case illustrates that appendicitis may be seen with typical HUS but this may represent inflammation due to the production of inflammatory mediators by Shiga-like toxin, rather than true primary appendicitis. The incidence of appendicitis following HUS is exceedingly rare but providers must have a high index of suspicion for gastrointestinal emergencies secondary to HUS. As appendiceal inflammation may be selflimited, this limits the diagnostic utility of an appendix ultrasound in HUS patients with abdominal pain.

\section{REFERENCES}

1.Whitington PF, Friedman AL, Chesney RW. Gastrointestinal disease in the hemolytic-uremic syndrome. Gastroenterology 1979;76:728-33.

2.Edmonson MB, Chesney RW. Hemolytic-uremic syndrome confused with acute appendicitis. Arch Surg. 1978;113(6):754-5.

3.Tune BM, Leavitt TJ, Gribble TJ, et al. The hemolyticuremic syndrome in California: A review of 28 nonheparinized cases with long-term follow-up. J Pediatr. 1973;82(2):304-310.

4.De la hunt $\mathrm{MN}$, Morris $\mathrm{KP}$, Coulthard $\mathrm{MG}$, et al. Oesophageal and severe gut involvement in the haemolytic uraemic syndrome. Br J Surg. 1991;78(12):1469-72. 
5.Fenton SJ, Kastenmeier A, Pysher TJ, et al. Acute appendicitis in a patient with hemolytic uremic syndrome: an unusual clinical scenario. Pediatr Surg Int. 2008;24(4):43941.

6.Jokiranta TS. HUS and atypical HUS. Blood. 2017;129(21):2847-2856.

7.Mayer CL, Leibowitz CS, Kurosawa S, et al. Shiga toxins and the pathophysiology of hemolytic uremic syndrome in humans and animals. Toxins (Basel). 2012;4(11):1261-87.

8.Talarico V, Aloe M, Monzani A, et al. Hemolytic uremic syndrome in children. Minerva Pediatr. 2016;68(6):441-455.

9.Siegler RL. Spectrum of extrarenal involvement in postdiarrheal hemolytic-uremic syndrome. J Pediatr. 1994;125(4):511-8.

Funding: None Declared

Conflict of Interest: None Declared

Corresponding Author: Shahid Nadeem, MD

University of Texas Southwestern Medical Center, Pediatric

Emergency Medicine, Dallas United States Shahid.Nadeem@UTSouthwestern.edu 